\title{
A Piezoelectric Minirheometer for Measuring the Viscosity of Polymer Microsamples
}

\author{
Almudena M. Sánchez, Roberto Prieto, Member, IEEE, Manuel Laso, and Teresa Riesgo, Member, IEEE
}

\begin{abstract}
This paper describes the electromechanical design, operating principles and performance of a rheometer able to characterize the rheological behavior of microsamples of viscoelastic materials, such as polymer solutions, melt, and rubbers. It was developed with a view to portability, robustness, and ease of operation for very small samples. The rheometer operates by subjecting the samples to small-amplitude sinusoidal strain rates via an inverse piezoelectric actuator and detecting the stress response of the material via a direct piezoelectric sensor. The device operates under frequency-sweep mode in a very wide range of frequencies. Required sample sizes are typically three orders of magnitude smaller than for conventional rheometers. Owing to its lack of moving parts, the rheometer has an extremely simple design and is insensitive to vibration. Measurements on pressuresensitive adhesives and other polymeric systems are presented and validated against a standard cone-and-plate rheometer.
\end{abstract}

Index Terms-Actuator, gain measurement, piezoelectric material, rheology, sensor, viscosity.

\section{INTRODUCTION}

$\mathbf{R}$ HEOMETERS are instruments used to obtain a quantitative characterization of the properties which define the mechanical behavior of materials. They work by measuring the reaction of the material to a mechanical excitation at different frequencies. Rheometers are, at the moment, large instruments which need a big quantity of fluid to work (around $20 \mathrm{ml}$ ). The development of a minirheometer is highly significant for the industry, not only for the polymer-producing companies but also in biochemistry and biomedicine fields, because it allows one to do a quick screening of the new synthesized components and also to have a portable instrument.

This paper describes the modeling, design and experimental validation of a new rheometer: the piezoelectric minirheometer. The piezoelectric minirheometer characterizes a polymer by exciting it mechanically through piezoelectric transducers, [1], [2]. There are many configurations of the piezoelectric transducers and the polymer sample. As an example, two of them are shown in Figs. 1 and 2.

In this paper, it will be analyzed which of them is the most suitable by combining the results of an analytical 1-D model and finite-element analysis (FEA). Analytical 1-D models of each part of the piezoelectric minirheometer are integrated in order to obtain a 1-D global model. Results of these models are

Manuscript received December 5, 2005; revised October 2, 2007.

The authors are with the Centro de Electrónica Industrial, Universidad Politécnica de Madrid, 28006 Madrid, Spain (e-mail: roberto.prieto@upm.es).

Color versions of one or more of the figures in this paper are available online at http://ieeexplore.ieee.org.

Digital Object Identifier 10.1109/TIE.2007.910528

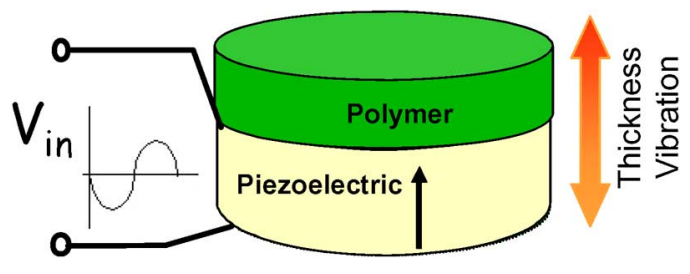

Fig. 1. Simplest configuration of the piezoelectric minirheometer.

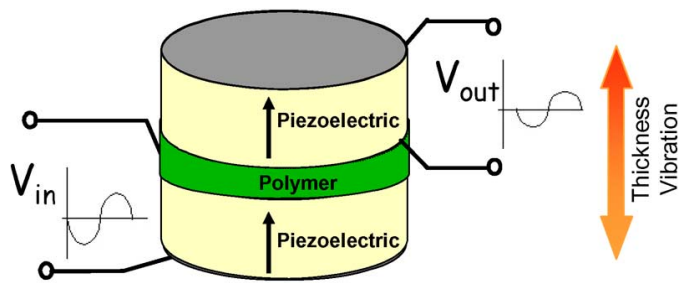

Fig. 2. Another possible configuration of the piezoelectric minirheometer.

complemented with 2-D/3-D information of FEA, providing an optimization of the piezoelectric minirheometer design. Several rheological sample properties, such as viscosity, can be derived from the comparison between the input and output electrical signals of the piezoelectric transducers, over a wide frequency range.

The main advantage of the piezoelectric minirheometer, with respect to conventional rheometers, is the reduction in more than three orders of magnitude of polymer quantity needed to do the measurements. The piezoelectric minirheometer needs very little fluid samples of less than $10 \mu \mathrm{l}$ and, from this point of view, it could be called "microrheometer" instead of minirheometer. There is no rheometer in the market able to do measurements with so little a polymer sample. Recently, the company GBC Scientific Equipment has commercialized a new rheometer, the Micro Fourier Rheometer [3], which needs samples (less than $100 \mu \mathrm{l}$ ) but not so small as the piezoelectric rheometer ones. In addition, the operation principle is different, and there are no piezoelectric material but mechanical mobile parts, which increase the global size of the system. In the state of the art, a rheometer made of piezoelectric transducers has been found [4], but it works in a different vibration mode and only gives a value of the viscosity at one frequency: the resonance frequency. It is not for polymers but liquids, and it is not a minirheometer because it needs a lot quantity of sample: a piezoelectric disc transducer is submerged in the liquid to do the measurements. Finally, there is another interesting rheometer, in researching state, made of piezoelectric transducers [5], [6], with the same basic operation principle of the piezoelectric 
minirheometer proposed in this paper (with the configuration of Fig. 2). The difference is that, in the rheometer in [5] and [6], two glasses are included between the sample and the piezoelectric transducers in order to support the sample. With the piezoelectric minirheometer, the support glasses are suppressed, and the piezoelectric behaves as a support for the sample as well as a transducer, reducing the final size of the global system. This also allows one to simplify the conditioning and amplification electronic needed to excite the polymer sample. Very recently, a flexure-based microgap rheometer (FMR) has been developed in [7]. The FMR is probably the only device with comparable sensitivity to handle microsamples. It yields visual structural information of fluid microstructure during the measurement in addition to the rheological characterization. It requires, however, very bulky ancillary equipment and is hardly portable. The minirheometer to be described was developed with a view to portability, robustness, and ease of operation for very small samples. In these respects, we believe it to be a unique instrument.

\section{Minirheometer Structure Selection}

Our application of the piezoelectric transducers to rheological characterization of polymers is a new concept that, first of all, must be validated, and models are very useful for this. In the design of the piezoelectric minirheometer, a lot of degrees of freedom must be determined: How to place the polymer in the piezoelectric transducers, how many transducers must be used (one that is the sensor and the actuator, two as a sensor and an actuator), how the dimensions and layer distribution of the transducers are, what the electrical signal to apply and to measure is ...

In order to test different possibilities, and considering that the custom-made samples of piezoelectric transducers are expensive, the use of models is completely justified to optimize them. Therefore, it is necessary to find a model that allows one to consider all the degrees of freedom of the piezoelectric minirheometer design.

\section{A. Minirheometer Model}

In this section, an analytical model for the whole minirheometer is developed by modeling the piezoelectric material and the polymer sample and then connecting both models, considering also the way to fix them. This model can be easily implemented in an electrical simulator (such as Simplorer, Pspice, ...), and it is very useful for the design stage, which is presented in Section II-B1.

1) Piezoelectric Model: Analytical equations which describe the piezoelectric transducers' behavior are difficult to solve as a 3-D system, as prior work shows [8]. Therefore, a simplification is done, considering the vibration of the piezoelectric in only one direction, the thickness direction, although, displacements in other directions may exist (the resultant model is an analytical 1-D model).

Therefore, piezoelectric transducers have been designed with the aid of an analytical 1-D model similar to the one used for the piezoelectric transformers in [9]. Since the model is based

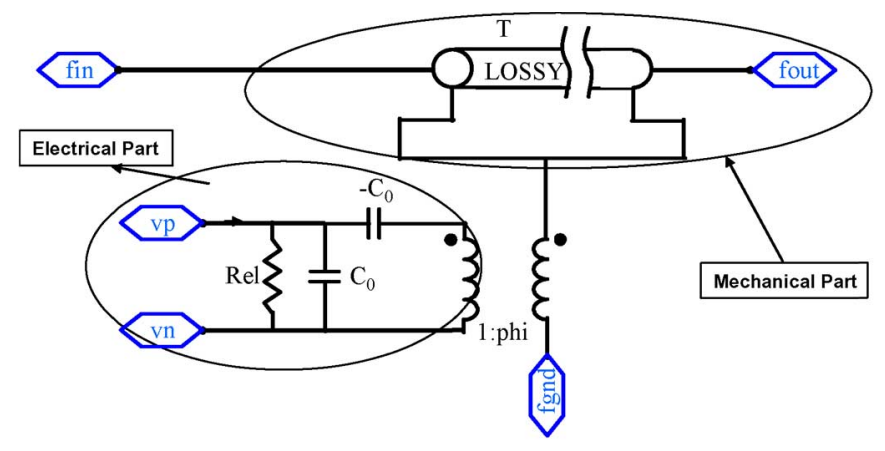

(a)

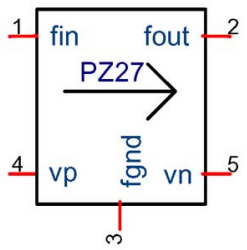

(b)

Fig. 3. One-dimensional piezoelectric transmission-line-based model. (a) Equivalent circuit. (b) Piezoelectric symbol.

in transmission lines, it takes into consideration all the vibration orders of the main frequency of resonance and not only the vicinity of the main frequency of resonance. This model allows the analysis of the frequency response of the system when the vibration is in the thickness direction. But, the model does not take into account the spurious vibration modes. Therefore, the vibration should be clean of spurious modes in order to extract conclusion using this model.

The electrical equivalent circuit is shown in Fig. 3(a) and the symbol of the model in Fig. 3(b). The reason in selecting this model is because it has not only electrical terminals (nodes vp and vn) but also mechanical terminals (nodes fin and fout), that are useful to study the influence of the connection between the piezoelectric transducers and the polymer. The transformer and negative capacitor $(-\mathrm{C} 0)$ stand for the electromechanical coupling between electrical and mechanical parts. The transmission line represents the mechanical behavior. The capacitance between electrodes is modeled with a capacitor $(\mathrm{C} 0)$ and the parallel resistor (Rel) takes into account the dielectric losses.

2) Polymer Model: The polymer model is based on an identification of mechanical variables with electrical variables in order to be able to implement the polymer model with the piezoelectric model in the same electrical simulator.

In the initial model [Fig. 4(a)], the inductors symbolize the polymer mass, the capacitor is inversely proportional to Young's modulus, and the resistor is our unknown, the viscosity $(\eta)$. But, the viscosity depends on the frequency (this is the effect to be measured with the minirheometer). Therefore, it is necessary to improve the model to take this effect into account with a voltage-controlled current source, which represents a frequency-dependant resistor [Fig. 4(b)].

3) Fixing Model: Piezoelectric transducers' performance depends strongly on the way of fixing it. Therefore, it is necessary to be very careful with their models. Several different 


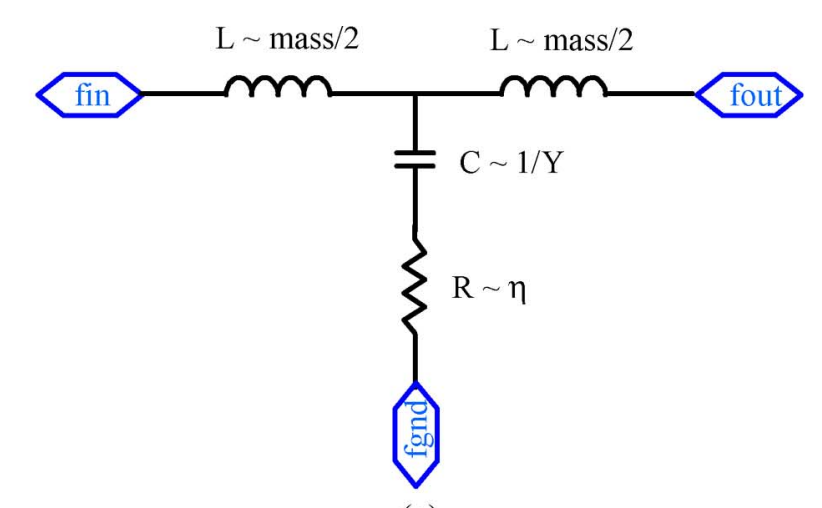

(a)

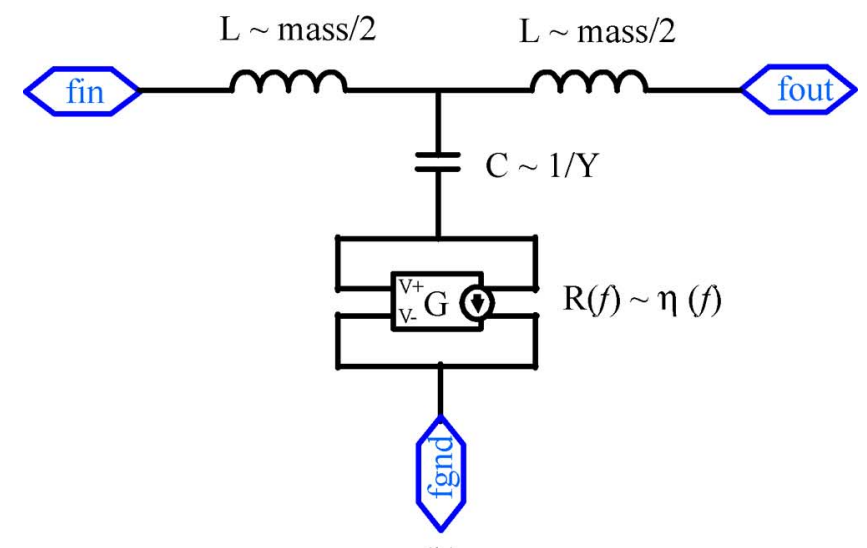

(b)

Fig. 4. Equivalent circuit of 1-D polymer model. (a) Initial model. (b) Improved model.

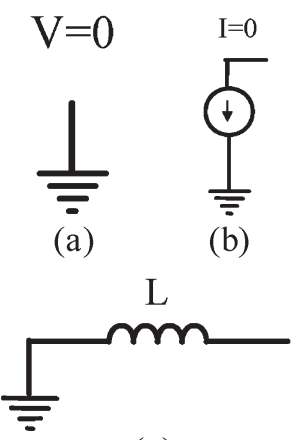

(c)

Fig. 5. Different models of the fixing. (a) Free node. (b) Totally fixed node. (c) Fixed node with a mass.

situations have been considered in the minirheometer design as follows.

1) Free node, represented by a zero-voltage source [Fig. 5(a)].

2) Totally fixed node, represented by a zero-current source [Fig. 5(b)].

3) Fixed node with a mass, represented by an inductor [Fig. 5(c)].

4) Piezoelectric Minirheometer Global Model: Models of each component have mechanical terminals available to be connected among them in an easy way. For example, the global

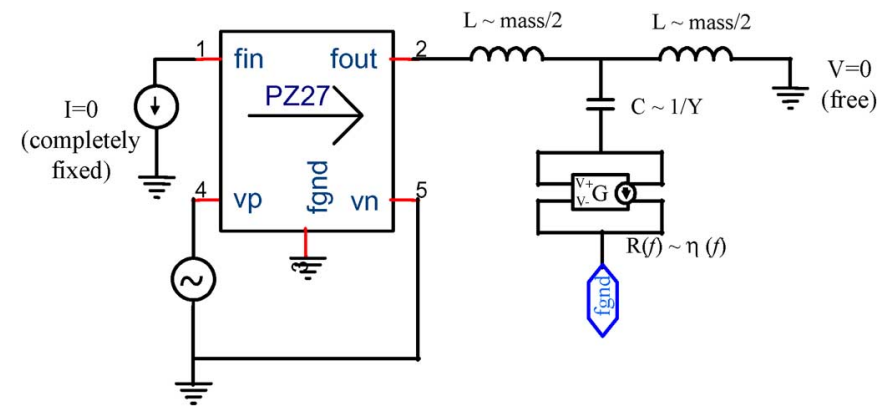

Fig. 6. Equivalent circuit of 1-D piezoelectric-minirheometer model with one end totally fixed and the other free.

model of the configuration shown in Fig. 1 is constructed by connecting properly the piezoelectric model and the polymer model, considering also the fixing of the external nodes (Fig. 6). In Fig. 7, the model for the configuration of Fig. 2 is detailed. Model generation is very quick because its input parameters are geometrical and material properties.

\section{B. Piezoelectric Minirheometer Design}

With the analytical 1-D model, it is possible to select the electrical signal to be measured (voltage gain, input impedance, etc.) and to design the sensor and actuator by selecting the type of material, thickness, and area. But, 1-D model does not take into account the 2-D/3-D geometry effects. Therefore, results of the 1-D model need to be complemented with 2-D/ 3-D information of FEA, providing an optimization of the piezoelectric transducers' design. The model has not been used for the full design of the piezoelectric transducers. The design has been completed using FEA tools in order to ensure that the vibration applied to the polymer is a thickness mode, checking the absence of spurious modes.

FEA tools are also very useful for considering practical aspects, such as the mechanical-fixing method, for the piezoelectrics or how to make the electrical connections and soldering.

1) Analysis and Selection of the Measuring Technique. 1-D Design: The main objective of using the 1-D model is to analyze different configurations of the minirheometer with the obtained 1-D model, determining the different electrical signals to be applied to the actuator and to be measured in the sensor. The selected signal has to be dependent on the viscosity of the polymer.

The simplest configuration has only one piezoelectric transducer that behaves as sensor and also as an actuator (Fig. 8). The polymer is placed over its surface. A sinusoidal voltage $V_{\text {in }}$ is applied to the electrodes and, by piezoelectric inverse effect, it vibrates. This vibration consists of compressions and expansions along the piezoelectric-actuator thickness. The vibration is then transmitted to the polymer. The selected shape of the piezoelectric is a disc, and the reasons for this selection will be justified in Section II-H. There is only one electrical signal to be measured: the input impedance as a function of the deformation rate (i.e., the operating frequency of the input voltage). But, the problem is that this electric signal does not present remarkable changes with the viscosity (Fig. 9). In this figure, obtained 


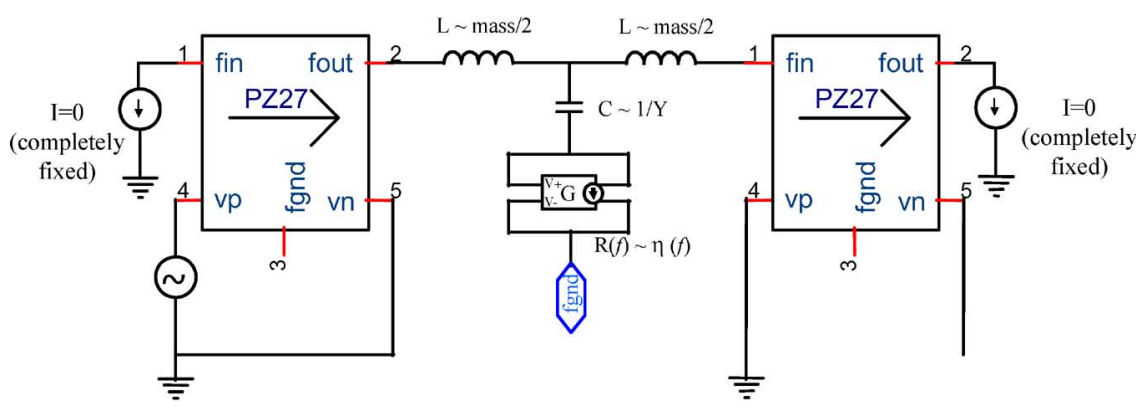

Fig. 7. Equivalent circuit of 1-D piezoelectric minirheometer with both ends totally fixed.

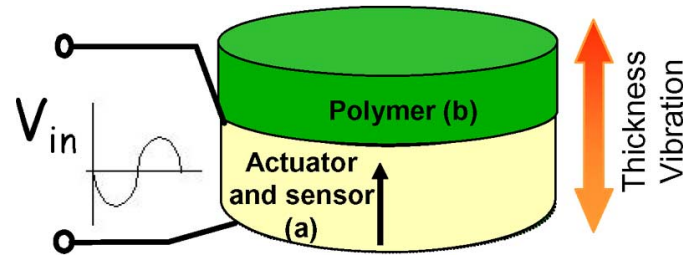

Fig. 8. Features of the simplest configuration of the piezoelectric minirheometer.

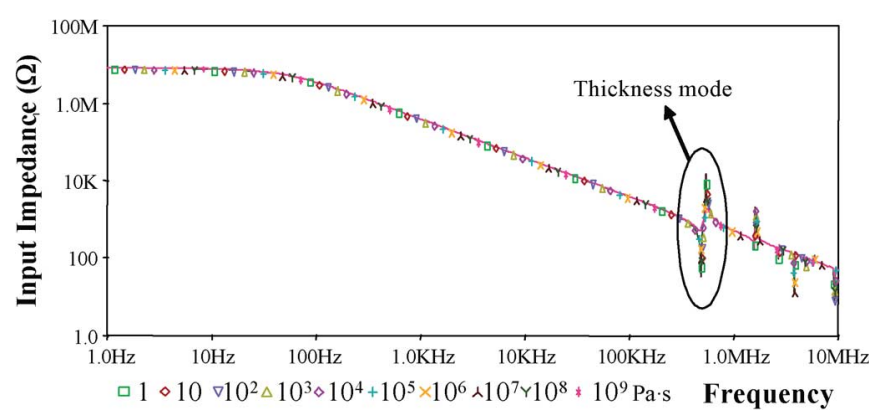

Fig. 9. Input impedance versus frequency for several polymers of different viscosity (from 1 to $10^{9} \mathrm{~Pa} \cdot \mathrm{s}$ ).

with the model described in Fig. 6, it represented the input impedance as a function of the frequency for several polymers of different viscosities. All the curves are superimposed in the whole frequency range, with the exception of the resonances. Nevertheless, it would be interesting to find an electrical signal that depends on the viscosity over a wide frequency range. Therefore, this configuration is rejected. This fact has also been validated with measurements in real samples.

The next option is to work with two transducers, one as a sensor (c) and the other as an actuator (a), both comprising a polymer (this is the configuration shown in Fig. 10). A sinusoidal voltage $V_{\text {in }}$ is applied to the electrodes of the actuator and a vibration is induced (by piezoelectric inverse effect), as in the previous configuration. Vibration is applied to the polymer sample, which transmits it to the piezoelectric sensor. The sensor transforms the mechanical vibration into a sinusoidal voltage $V_{\text {out }}$ (by piezoelectric direct effect), which has different amplitude than the input voltage and the phase shift. Two different options of electrical signal have been considered to be measured, using the model described in Fig. 7: input impedance of the actuator, as before, and output voltage in the sensor electrodes, calculating the voltage gain $\left(V_{\text {out }} / V_{\text {in }}\right)$. As in the previous case, the impedance does not allow one to discriminate

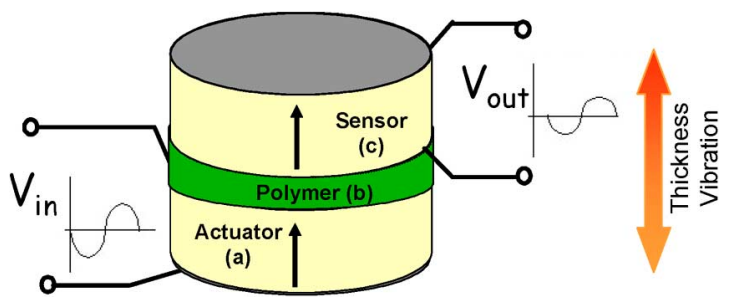

Fig. 10. Piezoelectric-minirheometer features.

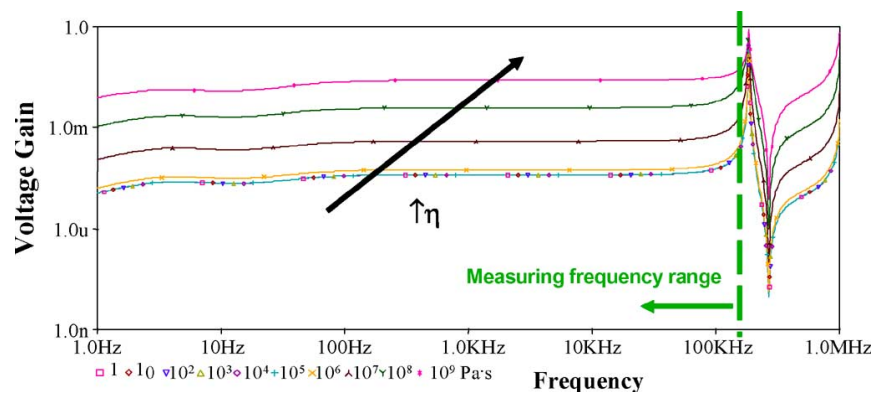

Fig. 11. Voltage gain versus frequency for several polymers of different viscosity (from 1 to $10^{9} \mathrm{~Pa} \cdot \mathrm{s}$ ).

different polymers. But the voltage gain changes with the viscosity, as it is shown in the graph of Fig. 11. At lower and medium frequencies (where the piezoelectric transducers work in a nonresonant mode), it is observed that the higher the polymer viscosity, the higher the voltage gain. The higher viscosity implies higher dissipation, and hence, an increase in voltage gain seems counterintuitive. However, when a more viscous fluid is placed between the plates, energy is transferred more efficiently from the actuator to the sensor, and there is no contradiction in this result. When the frequency increases and the first resonance appears, the curves for different viscosities becomes very close, so the measuring frequency range is limited by the first resonance frequency: The piezoelectric minirheometer works only in a nonresonant mode. Fig. 11 also shows the overlap of all curves up to $10^{5} \mathrm{~Pa} \cdot \mathrm{s}$, limiting the resolution of the system. Therefore, this minirheometer design will be able to measure the polymer viscosity only if it is higher than $10^{5} \mathrm{~Pa} \cdot \mathrm{s}$. Although this value is large if compared with viscosities of low-molecular-mass fluids, this value is at the low end of viscosities for polymer melts. Hence, it is not a serious limitation of the minirheometer.

Another important parameter to take into account in the design process is the fixing of the piezoelectric minirheometer. As it was said before, fixing is an important task to be considered 


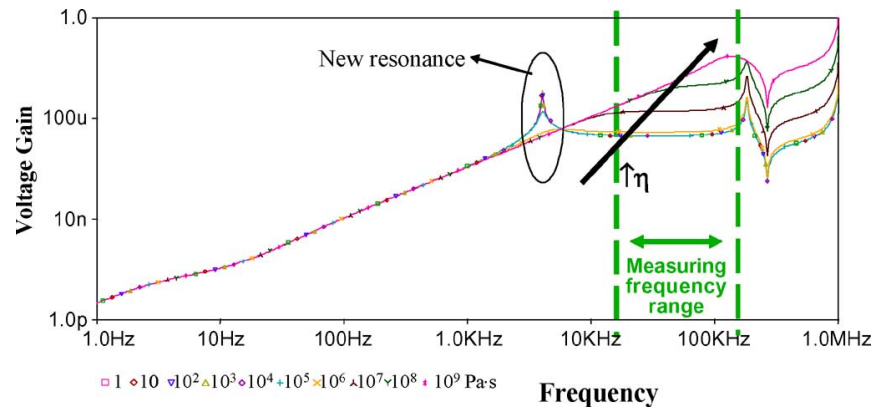

Fig. 12. Voltage gain versus frequency for several polymers of different viscosity (from 1 to $10^{9} \mathrm{~Pa} \cdot \mathrm{s}$ ).

in the design process, because the piezoelectric transducers are electromechanical devices, and the thickness vibration of the PT can be perturbed with the fixing. Voltage gain depends on the fixing, and it is maximum and more sensitive to the viscosity when both ends of the rheometer are totally fixed. In order to illustrate this, the case in which one of the ends of the piezoelectric minirheometer is left free has been simulated. Voltage-gain curves are shown in Fig. 12.

The main problem of this configuration is that the frequency range sensitive to the viscosity changes is reduced. The minirheometer could not work at low frequencies, because the curves are superimposed and a new resonance mode has appeared due to the new fixing conditions. Then, the mechanical conditions selected are with both ends totally fixed.

2) Piezoelectric Minirheometer 2-D/3-D Design: The onedimensional model fixes the piezoelectric area, but there are a lot of geometric possibilities in distributing this area, such as a disc, ring, plate, etc. FEA tools are useful to optimize the area distribution in the right shape. FEA tool selected is ATILA [10], because it is particularly developed for the simulation of the electromechanical coupling in 2-D/3-D of piezoelectric materials.

The simplest and cheaper shapes are the disc and the plate. The polymer film can be easily placed to be characterized in either of them. Therefore, both of them have been analyzed with the FEA tool. The main problem of the plate shapes is the border effect in the corners that can affect the force to be applied to the polymer (Fig. 13). Therefore, the geometry selected is a disc.

With the FEA tool, the spurious modes can also be tested. The spurious modes are resonances in different directions of the thickness dimension (nondesired vibrations). If these modes are excited, the piezoelectric actuator would make the polymer vibrate in a wrong way, and the characterization would not be reliable in these frequencies. Therefore, the measuring frequency range is determined by the spurious modes' position. Considering that the selected geometry for the piezoelectric is a disc, it is important to identify the position of the radial mode. This mode appears at lower frequencies than the thickness mode (because the resonance frequency is inversely proportional to the dimension of vibration) and will limit the working frequency range.

The FEA tool is also very useful for considering practical aspects, such as mechanical-mounting method for the piezoelectric transducers or how to make the electrical connections

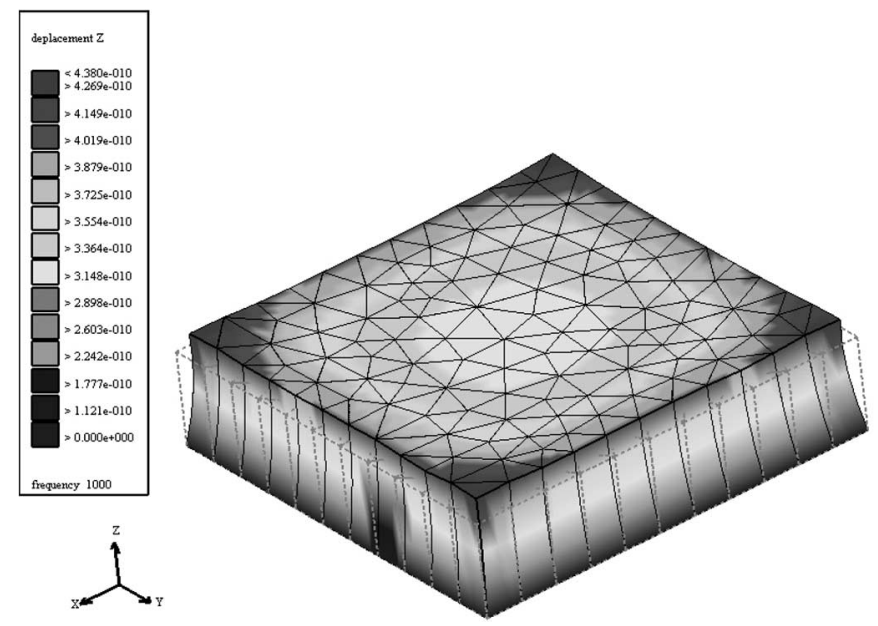

Fig. 13. Image extracted with the ATILA FEA tool of the mesh and displacement in the thickness dimension. Structure at rest drawn in dashed lines.

and soldering (3-D effects). The fixing method selected, with the upper and lower surfaces of the piezoelectric transducers totally fixed, allows the system to vibrate in the thickness direction in the whole measuring frequency range, as the simulation of Fig. 14 shows. It is necessary to highlight that, in this figure, the deformed shape is amplified to see the effect. The vibration is not an ideal thickness mode but very close to it, because the fixing influence is taken into account. Although the 1-D model does not provide information about the radial mode (or other spurious modes), it is valid in the frequency range selected for the minirheometer. In this range, the system presents a nonresonant mode in which, as checked with finite elements, the rheometer presents a thickness vibration.

3) Design Results: The piezoelectric transducers have been designed with the 1-D model to determine the type of material and dimensions (the thickness and total area) and, with the FEA tool, to determine the shape. Piezoelectric transducers are made of a lead-zirconate-titanate ceramic material (manufactured by Ferroperm Piezoceramics A/S [11]). The design criteria were mainly to minimize the transducers' size, maintaining the voltage gain high enough to be accurately measured. Besides, it is important to maximize the measuring frequency range, avoiding the spurious modes. Following all these criteria, the optimized piezoelectric minirheometer transducers' final design has been obtained. All its features have been summarized in Table I.

In Fig. 15, there are photographs of the piezoelectric samples [Fig. 15(a)], the prototype with the electrical connections and the polymer placed between the piezoelectrics [Fig. 15(b)], and the whole system with the mounting, two fixing plates joined by screws [Fig. 15(c)]. The precompression force exerted by the plates will have influence in the rheological behavior of the polymer sample, so it is necessary to guarantee that the mechanical conditions of the minirheometer are always the same. Therefore, the screws are tightened using a torque driver (Tohnichi RTD30CN) in order to assure a uniform contact between the polymer sample and the piezoelectric transducers. This also improves the reproducibility of the measurements, because the pressure of the fixing plates is always the same when different polymer samples are placed in the minirheometer. The 


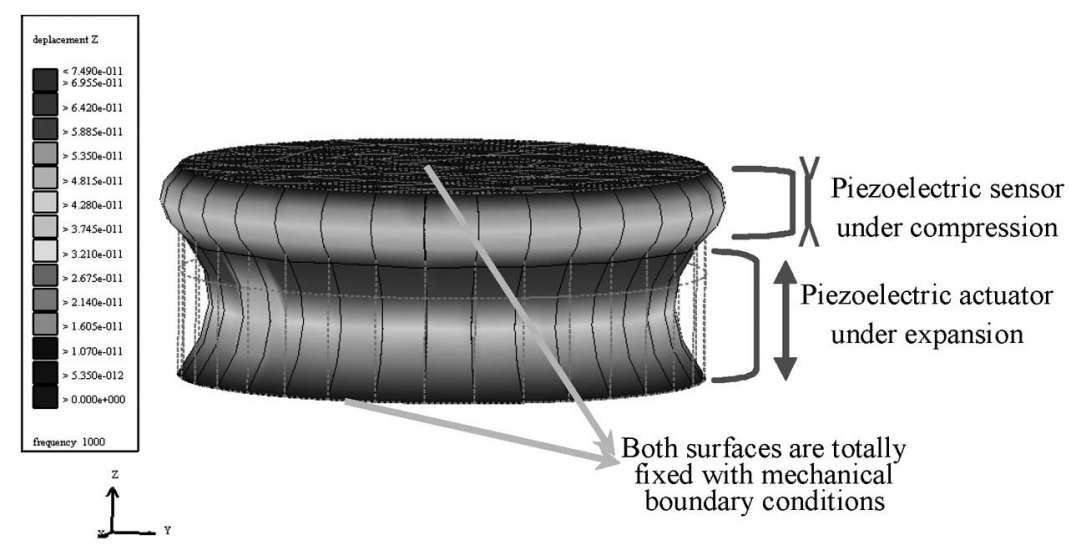

Fig. 14. Image extracted with the ATILA FEA tool of the mesh and displacement in the thickness dimension. Structure at rest drawn in dashed lines.

TABLE I

Piezoelectric-MiniRHeOMETER Design

\begin{tabular}{lc}
\hline Piezoelectric Material & Pz27 material [11] \\
\hline Piezoelectric Shape & Disc \\
\hline Piezoelectric Dimensions: & \\
\hline - Thickness & $2 \mathrm{~mm}$ \\
\hline - Diameter & $10 \mathrm{~mm}$ \\
\hline Mounting & Totally fixed \\
\hline Electrical signal to measure & Voltage gain \\
\hline
\end{tabular}

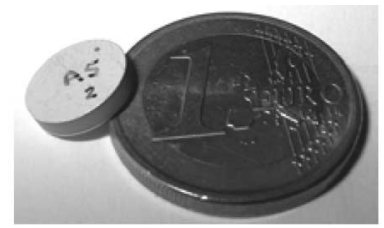

(a)

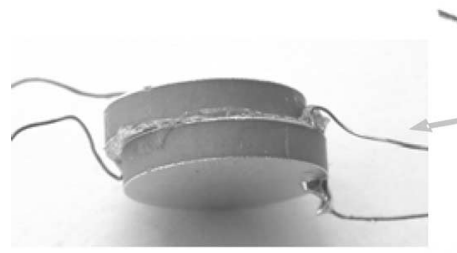

(b)

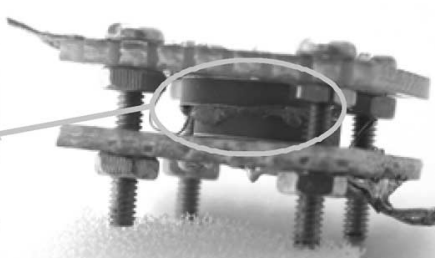

(c)
Fig. 15. Piezoelectric minirheometer prototype. (a) Transducer sample. (b) Prototype lateral view. (c) Fixing-method detail.

torque applied is not too high, trying to reduce the precompression force exerted by the plates. Since measurements were taken significantly longer times after prestressing the sample, neither residual stresses nor drift in the signal can possibly be expected.

It is important to emphasize that this is only the first prototype designed to validate the measurement principle. It will be necessary to improve the final appearance of the minirheometer to obtain an industrial application.

\section{Evaluation of Rheometrical MeAsurements IN THICKNESS MODE}

In this section, the proposed method to characterize the polymer viscosity through the electrical response of the piezoelectric transducers is evaluated.
The method will be validated measuring the viscosity of several polymer samples and comparing the results with the viscosity measurements obtained in a conventional rheometer (using a parallel-plate geometry). In this case, a simple shear flow field with spatially homogeneous velocity gradient is applied to the polymer. Therefore, it is not in contradiction with the use of viscosity in an extensional flow field, like the one used in the minirheometer. Viscosity is a general fluid property and is independent of flow geometry. At any rate, only one material property is required to describe the purely viscous behavior of the fluid (be it the shear or the extensional viscosity) and that is the only one that appears in the solution of any flow field, whether it corresponds to the propagation of shear waves, longitudinal waves, or any other more general flow situation.

\section{A. Analytical Relation Between the Minirheometer Voltage Gain and the Polymer Viscosity}

The piezoelectric minirheometer works in a nonresonantthickness mode, scanning the voltage from very low frequencies up to the first actuator resonance. Therefore, there is always linearity between electrical voltage and mechanical stress. Therefore, the polymer viscosity can be easily deduced from the voltage gain of the minirheometer $\left(G=V_{\text {out }} / V_{\text {in }}\right)$.

A procedure has been developed to find the relationship between the electrical signal [voltage gain as function of the frequency $f, G(f)]$ in the minirheometer and the mechanical property of the polymer (viscosity $\eta(f)$ ), which is the final objective. A simple equation will be deduced for this purpose.

First of all, it is necessary to find the relationship between the force applied to the polymer and its rheological properties. This is not difficult in a conventional rheometer where shear is the usual movement applied to the polymer sample. Shear 
constitutes a homogeneous problem where the deformation of the polymer is equal in the whole structure. In the minirheometer, the vibration of the piezoelectric is in the thickness direction, applying to the polymer a nonhomogeneous force (in terms of space and time). Therefore, it is not a trivial task to understand the behavior of the polymer under this force and to find the relationship between the force and the polymer viscosity. This problem has been considered in [12], using the Criminale-Ericksen-Filbey constitutive equation to describe the polymer reaction [13]. This is a good assumption that can be applied to simulate the behavior of more than $95 \%$ of the existing viscoelastic materials. In this paper, the process to achieve the required equation is not going to be detailed (it is explained in [12] step-by-step). It is only highlighted that the "quasi-steady-state" approximation used in the solution of the problem is totally valid for the specific case of the piezoelectric minirheometer because of the low amplitude of oscillation achieved with the piezoelectric transducers. The analytical-problem solution is restricted to the first order being, surprisingly, equal to the Newtonian solution. Therefore, the relationship between the polymer viscosity $\eta(f)$ and the applied force $F$, as a function of the frequency, has been simplified to

$$
F=k_{1} f \eta(f)
$$

where $k_{1}=\left(6 \pi^{2} R^{4} / h^{2}\right)$, where $R$ is the radius of the polymer sample (as the polymer is placed between two piezoelectric discs) and $h$ is the thickness of the polymer sample. This value of the viscosity is really a mean value, since the polymer viscosity under a compression vibration depends on its distance to the piezoelectrics, but it is enough to validate whether the piezoelectric minirheometer gives a correct measure of the viscosity. This analytical expression is essential to calculate the viscosity of a polymer placed in the minirheometer and to compare the result with the viscosity measurements done in a conventional rheometer.

In addition to that, as the piezoelectric minirheometer works in nonresonant mode, there is linearity between the voltages and the forces in the sensor and the actuator. Therefore, the vibration of the polymer implies a force over the piezoelectric sensor that is picked by this sensor and transformed to a voltage (proportional to the voltage gain of the minirheometer). This relation can be expressed as

$$
F=k_{2} \cdot G(f)
$$

where $k_{2}$ is the proportionality constant, dependent on geometrical parameters and material properties.

Combining (1) and (2) yields to

$$
\eta(f)=\frac{K \cdot G(f)}{f}
$$

where $K=\left(k_{2} / k_{1}\right)$.

Equation (3) can be applied for each frequency in the measuring range.

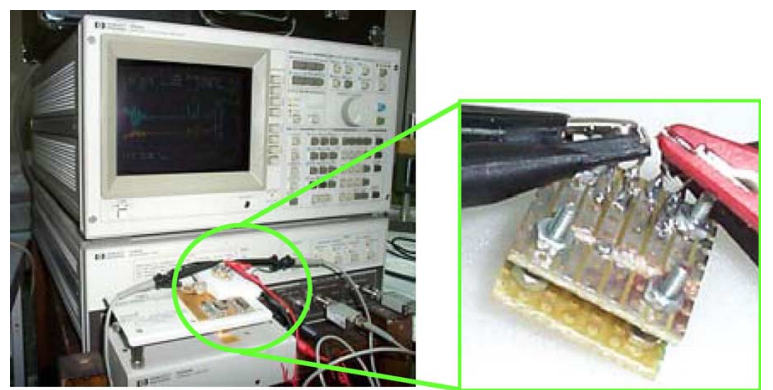

Fig. 16. Experimental setup. Piezoelectric-minirheometer prototype detail.

\section{B. Viscosity-Evaluation Method}

Once the relationship between gain and viscosity is extracted [(3)], the next stage is to describe the methodology developed to calculate the viscosity and validate the pattern. It has been divided into the following three steps.

\section{Step 1) Acquire a calibration pattern.}

A polymer of well-known viscosity is selected (it has been characterized with a conventional rheometer). The objective is to calculate the value of the $K$ constant. At one frequency $f_{1}$, the gain $G\left(f_{1}\right)$ is determined with the piezoelectric minirheometer, and it allows one to obtain $K$ with the following equation [obtained from (3)]:

$$
K=\frac{\eta\left(f_{1}\right) \cdot f_{1}}{G\left(f_{1}\right)} .
$$

\section{Step 2) Represent a plot of viscosity versus frequency.}

The same polymer is characterized for the rest of frequencies of the measuring frequency range with the piezoelectric microrheometer gain, and therefore, the viscosity is calculated with (3) and compared with the one obtained with a conventional rheometer.

It would be interesting to merge these two steps using the information of multiple frequencies to lead to a more accurate calculation of the parameter $K$. However, the number of polymer samples that were available for this paper was limited to a few. Therefore, working in two steps, it is possible to obtain a calibration pattern and validate the measurement principle with the same sample.

\section{Step 3) Validate the pattern.}

Other polymers are placed in the piezoelectric minirheometer to validate the calibration pattern [carrying out step 2) again].

The experimental results of these three steps are detailed in the next section.

\section{EXPERIMENTAL RESULTS}

\section{A. Experimental Setup}

The experimental setup used to measure the gain of the piezoelectric minirheometer is shown in Fig. 16. An impedance analyzer (HP-4194A) generates the input voltage and measures the output voltage, calculating the magnitude and phase of the voltage gain. 


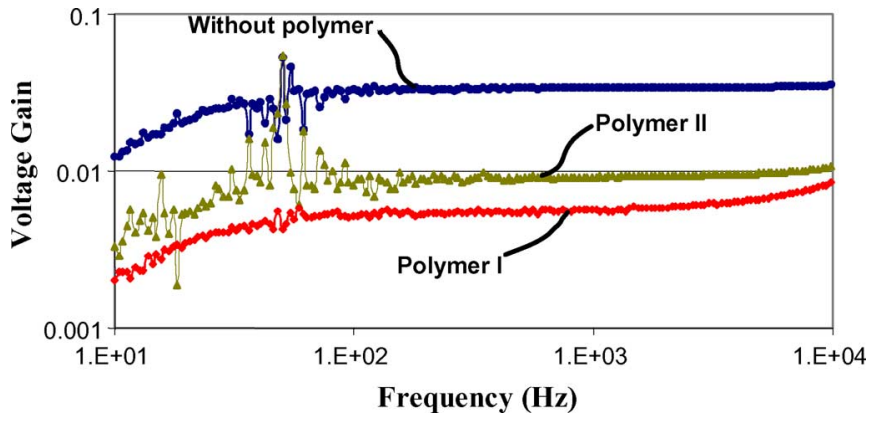

Fig. 17. Measures of voltage-gain magnitude versus frequency.

The piezoelectric minirheometer has been tested by measuring the viscosity of pressure-sensitive adhesive (PSA) polymers. These polymers have a non-Newtonian behavior; therefore, the viscosity depends on the deformation rate (frequency) or, in general, on the polymer-deformation history. All of the PSA samples provided by the manufacturer have the same thickness $(100 \mu \mathrm{m})$. Its area is determined by the piezoelectric transducer's shape, so the samples have been cut in a disc shape of $80 \mathrm{~mm}^{2}$, that means a very small volume of $8 \mu \mathrm{l}$. All the polymers have different viscosities, and for confidentiality reasons, their real names cannot be provided, so they have been called Polymer I, Polymer II, and Polymer III. Viscosity of Polymer I is the lowest and viscosity of Polymer II is the highest (Polymer III is at an intermediate stage).

As it was explained, the mechanical polymer property selected for the measurements is the viscosity. The polymers have been previously characterized with a conventional rheometer, and the resultant curves of viscosity versus frequency will be named as "theoretical" in order to be compared with the minirheometer ones. Therefore, a comparison between the piezoelectric minirheometer and conventional rheometer results will also be presented.

\section{B. Validation of the Measurement Technique}

First of all, it is necessary to verify if the minirheometer detects the presence of a polymer placed between both piezoelectric transducers, providing a detectable change in the voltage gain, which depends on the viscosity. Polymers I and II are placed to know if the precision of the minirheometer is enough to distinguish them.

As it was predicted by the simulations, voltage gain is suitable as a detector of the viscosity (Fig. 17). Polymers I and II are detected because gain drops with reference to the empty minirheometer gain. In addition, different polymers provide different gain so they can be distinguished: The higher the viscosity of the polymer, the higher its voltage gain (Polymer II viscosity is bigger than Polymer I viscosity).

The piezoelectric minirheometer model has been validated by comparing the voltage gain of the simulation with the results of measurements (Fig. 18). It is necessary to emphasize that the model of the fixing has been modified by considering that the plates do not provide the ideal fixation. The totally fixednode model has been adjusted with a parallel capacitor that takes into account the Young's modulus of plates. The model presents deviations but it satisfies all the requirements needed

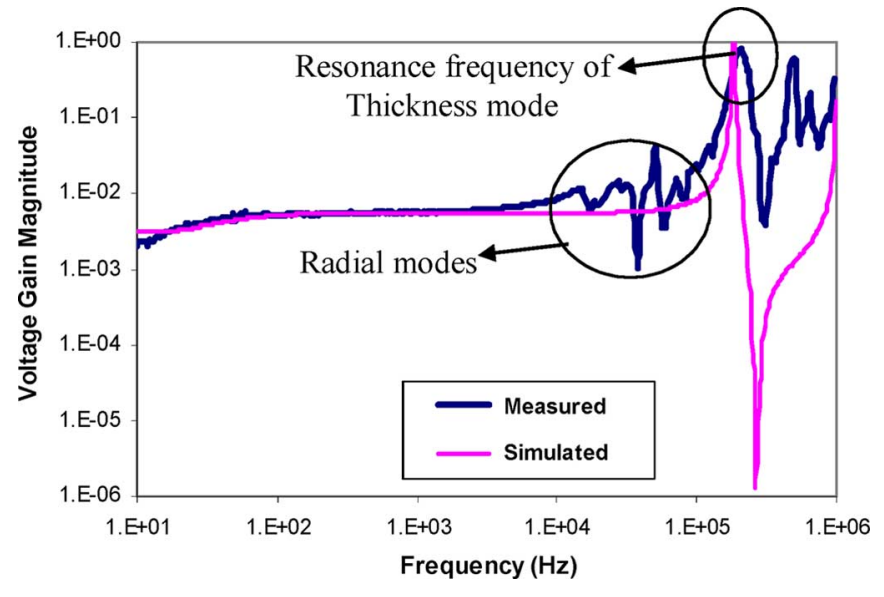

Fig. 18. Comparison between the simulated and measured gain.

in this specific application. It allows one to test that the voltage gain of the system depends on the viscosity. The resonance frequency of the thickness mode is also accurately predicted. Gain results above the thickness resonance are not valid, but it is not significant because this frequency range is out of the measuring range. The main deviation is in the frequency range from 10 to $100 \mathrm{kHz}$, where several resonances appear. They are radial modes of vibration that cannot be predicted with the 1-D model. Nevertheless, these modes are obtained with the FEA tool, as it has been previously detailed.

\section{Viscosity Experimental Results}

The piezoelectric minirheometer performance has been validated with all the polymer samples (Polymers I, II, and III). All the measurements were repeated with different samples of the same polymer in order to validate the reproducibility of the experimental measurements. The three steps of the evaluation method to obtain the viscosity of a polymer, as described earlier in Section III-B, are now illustrated with the measurement results of these three polymers.

\section{Step 1) Acquire a calibration pattern.}

The polymer selected as pattern for the piezoelectric minirheometer calibration is Polymer I, which is used to calculate the $K$ constant. The value of $K$ can be obtained from (4), selecting $f_{1}=498 \mathrm{~Hz}$ because it is the frequency where the gain of the minirheometer, in this particular case, does not have too much noise. Therefore, the voltage gain measured in the minirheometer is $G\left(f_{1}\right)=$ $4.14 \cdot 10^{-2}$.

This polymer was previously being characterized with a conventional rheometer and its "theoretical" viscosity is $\eta\left(f_{1}\right)=1680 \mathrm{~Pa} \cdot \mathrm{s}$.

By replacing all these values in (4), the constant $K$ is calculated $\left(K=2.02 \cdot 10^{7} \mathrm{~Pa}\right)$.

Therefore, the resultant calibration pattern is as follows:

$$
\eta(f)=\frac{2.02 \cdot 10^{7} G(f)}{f}
$$

which gives the viscosity in pascals per second if the frequency is in hertz. 


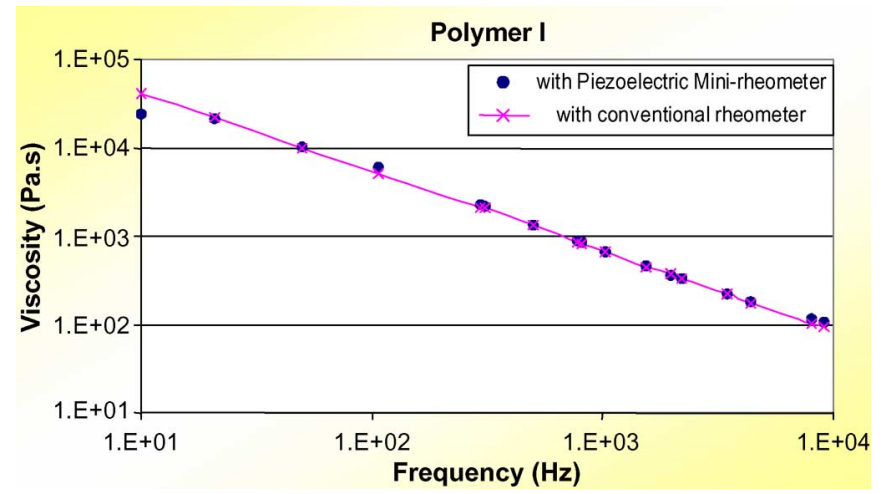

Fig. 19. Comparison between theoretical and experimental viscosity versus frequency of Polymer I. Both axes are in logarithmic scale.

\section{Step 2) Represent a plot of viscosity versus frequency.}

The calibration pattern [(5)] must be applied to the rest of frequencies, obtaining the viscosity in the whole measuring frequency range. In Fig. 19, a comparison is shown between the experimental results (dots), obtained with the pattern in the piezoelectric minirheometer, and the "theoretical" values (solid line), previously obtained with a conventional rheometer. The piezoelectric minirheometer is working properly because there is a good agreement between both results.

\section{Step 3) Validate the pattern.}

In order to complete the validation process, a second verification is done, placing other different polymer samples: Polymers II and III. The same as in (5), Polymer I is applied to obtain the viscosity from the voltage gain. As shown in Fig. 20(a), for Polymer II, and Fig. 20(b), for Polymer III, the piezoelectric minirheometer provides viscosity values which are very close to the expected ones. Therefore, it can be concluded that the pattern is valid and the working method is also correct.

\section{CONCLUSION}

In this paper, the piezoelectric minirheometer for the analysis of viscosity of polymers is modeled, designed, and validated. A prototype has been built and several polymer samples have been placed in it to measure the viscosity through the voltage gain. Therefore, a good agreement was observed between the curves of viscosity versus frequency obtained with the piezoelectric minirheometer and the "theoretical" curves, previously obtained with a conventional rheometer, for the three PSA polymers tested.

The main advantages of comparing with the commercial rheometers are the small size and wide measuring frequency range and, particularly, the little quantity of the polymer sample needed to do the measurements.

The commercial exploitation of the product has not been considered yet, although some companies have shown their interest in commercializing it. In order to obtain a portable system, the piezoelectric minirheometer should be independent of the impedance analyzer for the voltage-gain measurements. Therefore, the piezoelectric minirheometer is now being improved

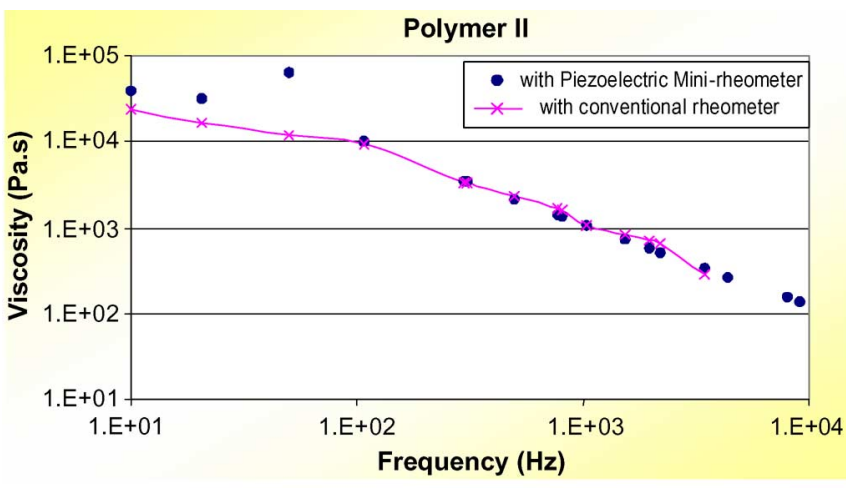

(a)

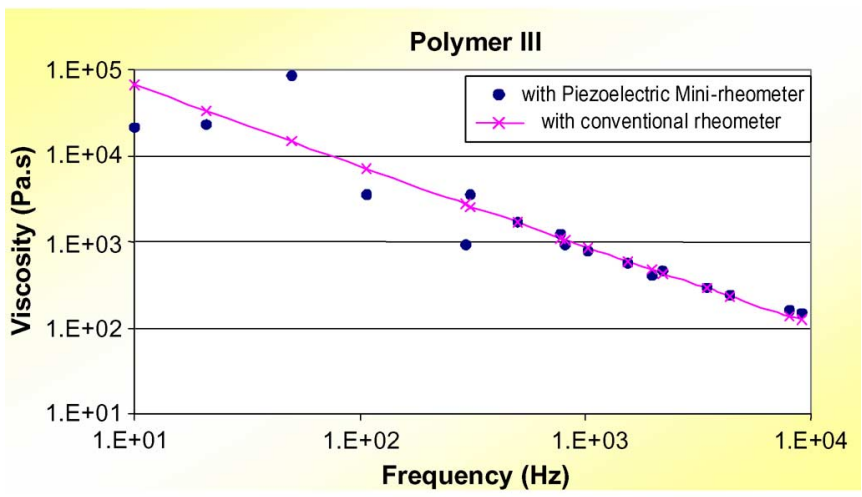

(b)

Fig. 20. Comparison between theoretical and experimental viscosity versus frequency. Both axes are in logarithmic scale. (a) Polymer II viscosity. (b) Polymer III viscosity.

with a chip [14], which contains the required electronics to control the actuator and the sensor, measuring the voltage gain in an autonomous way. For the integration, it is necessary to amplify and filter the output electrical signal of the piezoelectric minirheometer. The size of the whole system is expected to have about $3 \times 3 \mathrm{~cm}$ area and $1 \mathrm{~cm}$ height.

\section{REFERENCES}

[1] W. G. Cady, Piezoelectricity. New York: McGraw-Hill, 1946.

[2] IEEE Standard on Piezoelectricity, Jan. 29, 1988. ANSI/IEEE Std. 176-1987.

[3] Micro Fourier Rheometer MFR 2100. [Online]. Available: http://www. gbcsci.com/products/rheometer/mfr2100.asp

[4] K. Imano, R. Shimazaki, and S. Momozawa, "Measurement of viscosity of liquid using piezoceramic disk transducer with a radial expansion mode," IEICE Trans. Fundam., vol. E83-A, no. 1, p. 162, 2000.

[5] J. L. Gallani, L. Hilliou, P. Martinoty, F. Doublet, and M. Mauzac, "Mechanical behavior of side-chain liquid crystalline networks," J. Phys. II France, vol. 6, no. 3, pp. 443-452, Mar. 1996.

[6] J. Weilepp, J. J. Zanna, N. Assfalg, P. Stein, L. Hilliou, M. Mauzac, H. Finkelmann, H. R. Brand, and P. Martinoty, "Rheology of liquid crystalline elastomers in their isotropic and smectic A state," Macromolecules, vol. 32, no. 14, pp. 4566-4574, 1999.

[7] C. Clasen, B. P. Gearing, and G. H. McKinley, "The flexure-based microgap rheometer (FMR)," J. Rheology, vol. 50, no. 6, pp. 883-905, Nov./Dec. 2006.

[8] A. Iula, N. Lamberti, and M. Pappalardo, "An approximated 3-D model of cylinder-shaped piezoceramic elements for transducer design," IEEE Trans. Ultrason., Ferroelectr., Freq. Control, vol. 45, no. 4, pp. 1056 1064, Jul. 1998.

[9] J. A. Oliver, R. Prieto, M. Sanz, J. A. Cobos, and J. Uceda, "1D modeling of multi-layer piezoelectric transformers," in Proc. 32nd IEEE PESC, 2001, vol. 4, pp. 2097-2102. 
[10] ATILA. [Online]. Available: http://www.cedrat.com/software/atila/ atila.htm

[11] Ferroperm Piezoceramics A/S Catalog. [Online]. Available: http://www. ferroperm-piezo.com/

[12] R. Bird Byron et al., "Dynamics of polymeric liquids," in Fluid Mechanics, vol. 1. New York: Wiley, 1987.

[13] W. O. Criminale, Jr., J. L. Ericksen, and G. L. Filbey, Jr., "Steady shear flow of non-Newtonian fluids," Arch. Ration. Mech. Anal., vol. 1, no. 1, pp. 410-417, Jan. 1958 .

[14] A. de Castro, T. Riesgo, E. de la Torre, Y. Torroja, and J. Uceda, "Reusable and reconfigurable system for sensor interfacing and control," in Proc. DCIS Conf., Santander, Espanã, 2002, pp. 213-218.



Almudena M. Sánchez was born in Madrid, Spain, in 1976. She received the B.S. degree in physics from the Universidad Complutense of Madrid, Madrid, Spain, in 1999 and the Ph.D. degree in electrical engineering from the Universidad Politécnica de Madrid, Madrid, in 2006.

In 2000, she joined the Centro de Electrónica Industrial, Universidad Politécnica de Madrid, where she is currently working in the area of electrical engineering. Her research interests include modeling and design of piezoelectric transformers and piezoelectric transducers for rheological applications.



Roberto Prieto (M'99) was born in Madrid, Spain, in 1969. He received the M.Sc. and Ph.D. degrees in electronic engineering from the Technical University of Madrid, Madrid, in 1993 and 1998, respectively.

Since 1994, he has been an Assistant Professor with the Technical University of Madrid (Centro de Electrónica Industrial), where he is currently Associate Professor. He has published more than 90 papers in international conferences and journals, most of them from the IEEE. His research interests include high-frequency magnetic components, modeling of power-electronic systems and development of computer-aided design tools for power-electronic applications.

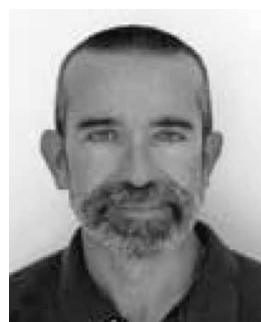

Manuel Laso received the M.Sc. degree in chemical engineering and the Dr.Sc. degree in materials science from the Institut für Integrierte Systeme, Eidgenoessische Technische Hochschule (ETH) Zurich, Zurich, Switzerland.

After working in industry for four years, he returned to ETH Zurich as a Senior Scientist. Since 1993, he has been a Professor of materials science with the Centro de Electrónica Industrial, Universidad Politécnica de Madrid, Madrid, Spain. He was a Visiting Professor with Massachusetts Institute of Technology, Cambridge, University of Cambridge, Cambridge, U.K., and University Erlangen-Nuremberg, Erlangen, Germany. He is the Author of approximately 60 papers with more that 700 citations by other authors. $\mathrm{He}$ is currently working on "Smart Materials Modelling," (John Wiley, to appear in 2008).

Dr. Laso was elected as Fellow with the Institute of Physics since 2004. He is a member of the Editorial Board of "Modelling and Simulation in Materials Science and Engineering." He is the President of the Scientific Advisory Board of Repsol-YPF. He is an active Consultant for Shell, Exxonmobil, Borealis, Rhodia, etc.

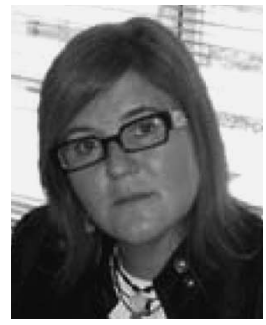

Teresa Riesgo (M'96) was born in Madrid, Spain, in 1965. She received the M.Sc. and Ph.D. degrees in engineering from the Universidad Politecnica de Madrid (UPM), Madrid, in 1989 and 1996, respectively.

Since 2003, she has been a Professor with the Centro de Electrónica Industrial, UPM. Her research interests are focused on embedded-system design, wireless-sensor networks, configurable systems, and power estimation in digital systems. She has published a large number of papers in this field and has participated and acted as main researcher in several European Union-funded projects. She is currently responsible for research and international relations at Escuela Técnica Superior de Ingenieros Industriales, one of the engineering schools of UPM. 